\title{
Blood stream infections in the first year after liver transplantation in children
}

\author{
Yeong Eun Kim${ }^{1}$, Seok Hee Oh${ }^{1}$, Ho Jung Choi ${ }^{1}$, Jung-Man Namgoong ${ }^{2}$, Dae Yeon Kim², Shin Hwang ${ }^{3}$, Sung Gyu Lee ${ }^{3}$, \\ Kyung Mo Kim ${ }^{1}$
}

\footnotetext{
${ }^{1}$ Department of Pediatrics-G-I/Hepatology, Asan Medical Center, University of Ulsan College of Medicine, Seoul, Korea

${ }^{2}$ Department of Surgery-Pediatric, Asan Medical Center, University of Ulsan College of Medicine, Seoul, Korea

${ }^{3}$ Department of Surgery, Asan Medical Center, University of Ulsan College of Medicine, Seoul, Korea
}

Background: Post-liver transplantation (LT) blood stream infection (BSI) is a major cause of death in both adult but pediatric LT. In this study, we aimed to analyze the profile of BSI according to the post-transplantation periods and determine the risk factors of the BSI within the first year after LT in our center.

Methods: Children under 18 years of age who underwent LTs at Asan Medical Center from December 1994 to June 2020 were retrospectively reviewed. BSI was defined based on conventional criteria from the Centers for Disease Control (CDC) guidelines with minor modifications. We used t-tests, Mann-Whitney U-test, chi-square test, and Fisher exact test in univariate analysis. The receiver operating characteristic (ROC) curve analysis and logistic regression analysis were used in the multivariate analysis to determine the risk factors of the BSI.

Results: During the study period, 378 children had primary LTs, consisted of 287 living donor liver transplantations (LDLTs; 76\%) and 91 deceased donor liver transplantations (DDLTs; 24\%). The median recipient age was 1.6 years (3 months-17.4 years) and the median weight was $10.9 \mathrm{~kg}(4-90 \mathrm{~kg})$. Biliary atresia (53\%) and acute liver failure (23\%) were the most common etiology for pediatric LTs. Among 378 patients received primary LTs, 106 patients $(28 \%)$ experienced pathogen-proven BSI during the first year after LT. Of them, 71 patients (67\%) had only one episode of BSI, while 35 patients (33\%) had more than one BSI. Most common identified organism coagulase-negative staphylococci, followed by Enterococcus spp. and Streptococcus spp., both in LDLT and DDLT groups. In multivariate analysis, younger age, liver support, longer hospital stay, portal vein complication, and growth failure were associated with increased risk of BSI.

Conclusions: BSI was frequently observed in pediatric LT and the BSI profile of pathogens may be informative to establish center's own policy against BSI after transplant.

Corresponding author: Seok Hee Oh

E-mail: seakhee.oh@amc.seoul.kr

(C) The Korean Society for Transplantation

This is an Open Access article distributed under the terms of the Creative Commons Attribution Non-Commercial License (http://creativecommons.org/licenses/by-nc/4.0/) which permits unrestricted non-commercial use, distribution, and reproduction in any medium, provided the original work is properly cited. 